This is an original manuscript of an article published by IEEE on May 2020 https://ieeexplore.ieee.org/abstract/document/9097101 DOI : https://doi.org/10.1109/ISEF45929.2019.9097101 


\title{
3D Finite Element Model using SIBC to Accelerate Electromagnetic Thermal Simulation of Induction Thermography Technique
}

\author{
Abdoulaye Ba, Huu-Kien Bui, Gérard Berthiau, Didier Trichet, Guillaume Wasselynck \\ University of Nantes \\ Institute of Research in Electrical \\ Energy of Nantes Atlantique \\ 44600 Saint-Nazaire Cedex, France \\ abdoulaye.ba@univ-nantes.fr
}

\begin{abstract}
We present a lightened 3D finite element model for coupled electromagnetic thermal simulation of the induction thermography non-destructive testing technique to reduce the computation time. The time harmonic electromagnetic problem is expressed in $A-\phi$ formulation and lightened by using the Surface Impedance Boundary Condition (SIBC) applied to both the massive induction coil surface and the surface of conductor workpiece under test including open cracks. The external circuit is taken into account by using the impressed voltage or the impressed current formulation

Index Terms-Impedance Boundary Condition, external electrical circuit, 3D FEM, coupled model, induction thermography
\end{abstract}

\section{INTRODUCTION}

In the modeling of induction thermography technique, the classical full finite element model with impressed external circuit constraints, such as current and voltage, is well adapted for the modeling of complex shape coil [2]. However, this model is very time consuming due to the necessity of fine mesh in the skin depth layer. We present in this paper an accurate lightened 3D finite element model for the coupled electromagnetic thermal simulation of the induction heating process using the Surface Impedance Boundary Condition (SIBC), is used in order to reduce the computation time.

\section{WEAK FORMULATION OF SIBC METHOD}

When using finite element with SIBC, the volume of the conductor has to be excluded from the finite element resolution, only the surface meshes are required. If all conductors are replaced by their surface, the Maxwell's equation to be solved is $\operatorname{curlH}=0$ in the remaining nonconducting domains and can be written in term of magnetic vector potential as:

$$
\operatorname{curl}\left(\frac{1}{\mu} \operatorname{curl} A\right)=0
$$

where the condition $\boldsymbol{A}=\left.0\right|_{\Gamma}$ is applied on the boundary $\Gamma$ of the surrounding air box. By applying the weighted residual method to the equation (1) with the test functions $\left(\mathbf{w}^{e_{i}}+\right.$ $\left.\operatorname{grad} w^{n_{i}}\right)$ where $\mathbf{w}^{e_{i}}$ and $w^{n_{i}}$ are respectively the edge and nodal shape function and by using Green's formulae, we obtain the weak formulation of the electromagnetic problem:

$$
\begin{aligned}
& \int_{\Omega} \boldsymbol{c u r l} \mathbf{w}^{e_{i}} \frac{1}{\mu} \operatorname{curl} \boldsymbol{A} d v \\
& -\int_{\Gamma^{c}}\left(\mathbf{w}^{e_{i}}+\boldsymbol{g r a d} w^{n_{i}}\right)(\boldsymbol{n} \times \boldsymbol{H}) d s=0
\end{aligned}
$$

where $\boldsymbol{n}$ is the outward normal vector of $\Gamma_{c}=\Gamma_{c_{1}} \cup \Gamma_{c_{2}}$ with $\Gamma_{c_{1}}$ and $\Gamma_{c_{2}}$ are the whole bounded boundary of the workpiece and the coil, and $\boldsymbol{A}$ discretized by edge element. On the surface of the conductors $\Gamma_{c}$, one applies the SIBC [3]:

$$
\boldsymbol{n} \times\left.\boldsymbol{H}\right|_{\Gamma^{c}}=\boldsymbol{n} \times\left.\frac{1}{\mu} \boldsymbol{c u r l} \boldsymbol{A}\right|_{\Gamma^{c}}=\frac{1}{Z_{c}}(\boldsymbol{n} \times \boldsymbol{E}) \times\left.\boldsymbol{n}\right|_{\Gamma^{c}}
$$

where $Z_{c}$ is the surface impedance which depends on the conductor properties. In the literature, three expressions of the surface impedance $Z_{c}$ known respectively as Leontovich, Mitzner and Rytov approximation can be given. The Leontovich approximation [5] is $Z_{c}^{\text {Leontovich }}=(1+j) /(\sigma \delta)$ with $\delta=\sqrt{2 /(\omega \sigma \mu)}$ where $\omega=2 \pi f$ is the angular frequency, $j$ the imaginary unit.

As a result, the equations of $\boldsymbol{A}-\phi$ weak formulation with SIBC method when a voltage $\mathrm{V}$ is impressed between the two conductors of the coil end on which the potential is considered constant, reads :

$$
\begin{aligned}
& \int_{\Omega} \boldsymbol{\operatorname { c u r l }} \mathbf{w}^{e_{i}} \frac{1}{\mu} \boldsymbol{c u r l} \boldsymbol{A} d v+\int_{\Gamma^{c}}\left(\boldsymbol{n} \times \mathbf{w}^{e_{i}}\right) \frac{1}{Z_{c}}(\boldsymbol{n} \times j w \boldsymbol{A}) d s \\
& +\int_{\Gamma^{c}}\left(\boldsymbol{n} \times \mathbf{w}^{e_{i}}\right) \frac{1}{Z_{c}}\left(\boldsymbol{n} \times j w \boldsymbol{g r a d} \phi^{f_{d y n a m i c}}\right) d s \\
& =-\int_{\Gamma^{c}}\left(\boldsymbol{n} \times \mathbf{w}^{e_{i}}\right) \frac{1}{Z_{c}}\left(\boldsymbol{n} \times j w \boldsymbol{g r a d} \alpha \phi^{i}\right) d s \\
& \quad \int_{\Gamma^{c}}\left(\boldsymbol{n} \times \boldsymbol{g r a d} w^{n_{i}}\right) \frac{1}{Z_{c}}(\boldsymbol{n} \times j w \boldsymbol{A}) d s \\
& +\int_{\Gamma^{c}}\left(\boldsymbol{n} \times \boldsymbol{g r a d} w^{n_{i}}\right) \frac{1}{Z_{c}}\left(\boldsymbol{n} \times j w \boldsymbol{g r a d} \phi^{f_{d y n a m i c}}\right) d s \\
& =-\int_{\Gamma^{c}}\left(\boldsymbol{n} \times \boldsymbol{g r a d} w^{n_{i}}\right) \frac{1}{Z_{c}}\left(\boldsymbol{n} \times j w \boldsymbol{g r a d} \alpha \phi^{i}\right) d s
\end{aligned}
$$

978-1-7281-1560-3/19/\$31.00 @2019 IEEE 


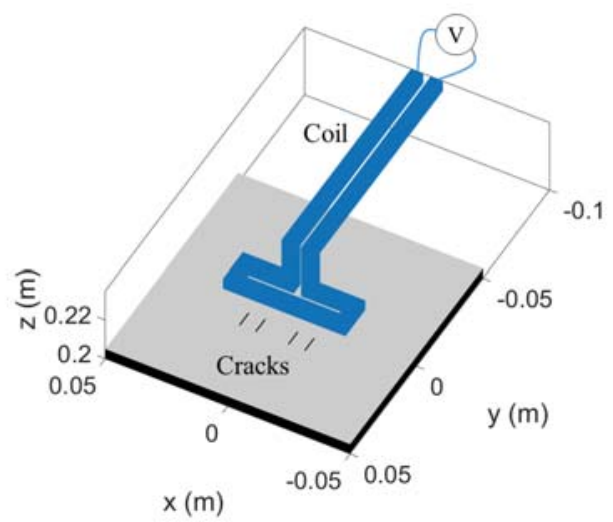

Fig. 1. Induction thermography NDT test case.

TABLE I

COMPARISON BETWEEN FULL-FEM AND SIBC-FEM SYSTEM.

\begin{tabular}{|l|c|c|}
\hline & Full-FEM & SIBC-FEM \\
\hline Total number of edge unknowns & 1505300 & 964135 \\
Total number of node unknowns & 166443 & 36487 \\
\hline Matrix assembly time $(s)$ & 86 & 31 \\
Matrix resolution time $(s)$ & 3374 & 508 \\
\hline
\end{tabular}

with $\alpha$ computed for all nodes of the coil surface [4]. The function $\alpha$ is equal to 1 on $\Gamma_{c_{2}}$ and equal to 0 on $\Gamma_{c_{1}}$. In SIBC method, the current can be computed as :

$$
I=\int_{\Gamma_{c_{2}}}(\boldsymbol{n} \times \boldsymbol{g} \boldsymbol{r a d} \alpha) \frac{1}{Z_{c}}(\boldsymbol{n} \times j w(\boldsymbol{A}+\boldsymbol{g r a d} \phi)) d s
$$

\section{ApPLICATION to INDUCTION THERMOGRAPHY NDT}

The configuration in the Fig.1 is used to simulate the electromagnetic thermal model with SIBC method. The workpiece has four thin open cracks and an $\mathrm{AC} 10 \mathrm{~V}$ voltage of $10 \mathrm{kHz}$ is ompressed between the ends of the coil. The workpiece is heated during 1 second. The temperature evolution is observed on the upper surface of the worpiece. Two following methods are compared:

- The classical full finite element method (Full-FEM)

- The SIBC finite element with impressed voltage with Leontovich's impedance (SIBC-FEM)

The Table. I gives comparisons between the Full-FEM system and the SIBC-FEM system. Note that the resolution time for thermal problem for the two model is practically identical. The resolution time in the Table. I is given for the electromagnetic problem. In the present test case, the deviation is not higher than $7 \%$ and the computation time is 6.6 times lower than the classical model.

\section{CONCLUSiOn}

The SIBC can be used to alleviate 3D finite element models for problem with presence of massive coils and workpieces with open cracks. The accuracy and the usefulness of the method for the design of the induction thermography NDT technique have been shown with acceptable deviation compared with a full FEM model.

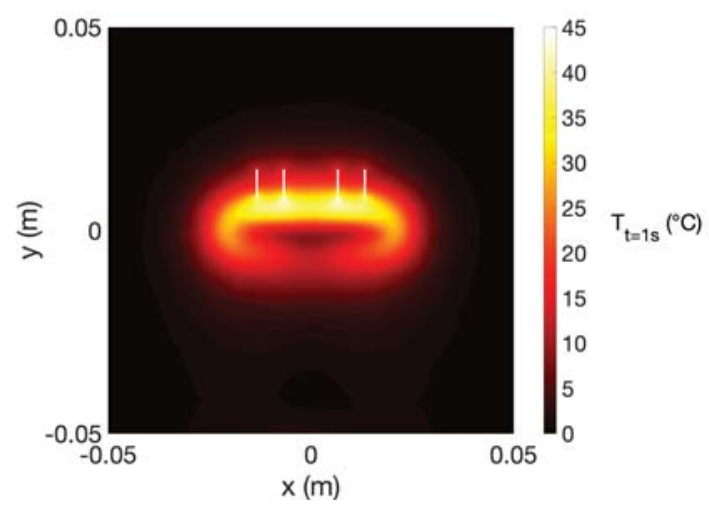

Fig. 2. Temperature distribution on the surface of the workpiece at $t=1 \mathrm{~s}$ computed with Full-FEM model.

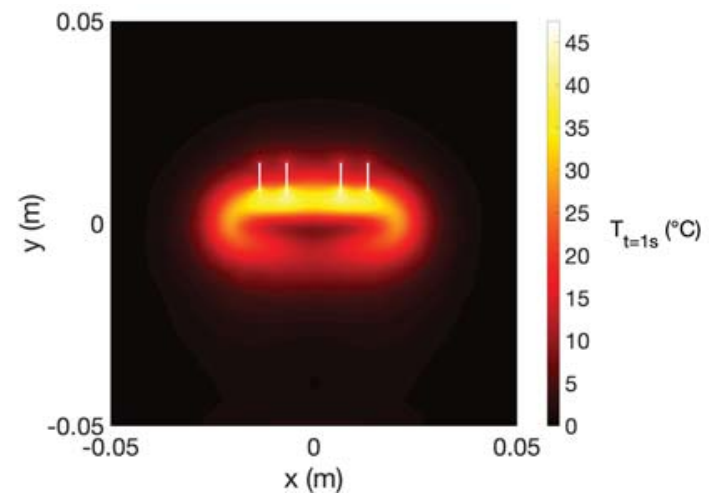

Fig. 3. Temperature distribution on the surface of the workpiece at $t=1 \mathrm{~s}$ computed with SIBC-FEM model.

\section{ACKNOWLEDGMENT}

This project has received funding from the European Union's Horizon 2020 research and innovation programme under the Marie Sklodowska-Curie grant agreement No 722134NDTonAIR.

\section{REFERENCES}

[1] M. He, L. Zhang, W. Zheng, and Y. Feng, "Investigation on a new inducer of pulsed eddy current thermography," AIP Advances, 2016, vol. 6, no 9, p. 095221 .

[2] H. K. Bui, G. Wasselynck, D. Trichet and G. Berthiau, "Performance assessment of induction thermography technique applied to carbon-fiberreinforced polymer material," IEEE Transactions on Magnetics, vol. 51, 2015.

[3] A. Desmoort, Z. De Grève, P. Dular, C. Geuzaine and O. Deblecker, "Surface Impedance Boundary Condition With Circuit Coupling for the 3-D Finite-Element Modeling of Wireless Power Transfer,' IEEE Transactions on Magnetics, vol. 53, 2017.

[4] T. Henneron, S. Clénet, and F. Piriou, "Calculation of extra copper losses with imposed current magnetodynamic formulations," IEEE Transactions on Magnetics, vol. 42, no 4, p. 767-770, 2006.

[5] S. Yuferev, and N. Ida, "Selection of the surface impedance boundary conditions for a given problem," IEEE Transactions on Magnetics, vol. $35,1999$. 